LES REPRÉSENTATIONS SOCIALES DE LA GROSSESSE À L’ADOLESCENCE

\title{
LES REPRÉSENTATIONS SOCIALES DE LA GROSSESSE À L'ADOLESCENCE: DES PRISES DE POSITION DOMINANTES ET D'AUTRES ... DÉSTABILISANTES
}

\author{
FRANCINE DUFORT, KATHLEEN BOUCHER, \\ Université Laval \\ ÉDITH GUILBERT, \\ Direction de la santé publique de Québec et Université Laval \\ LOUISE SAINT-LAURENT et LAURENCE FORTIN-PELLERIN \\ Université Laval
}

RÉSUMÉ

\begin{abstract}
Cette étude porte sur les représentations sociales de la grossesse à l'adolescence. Dix-huit focus groups sont menés auprès de 150 filles et garçons, âgés de 15 à 17 ans. Une analyse de contenu qualitative met en relief 4 dimensions (émotive, réflexive, psychobiologique et économico-sociale) et 4 prises de position (négative, positive, ambivalente, dynamique), autour desquelles s'organisent 4 types de significations accordées à la grossesse à l'adolescence: la grossesseproblème, la grossesse-projet, la grossesse-tension et la grossesse-pouvoir. Quelques avenues en matière de pratiques éducatives sont abordées, notamment l'importance d'aller au-delà des messages alarmistes, d'instaurer un dialogue ouvert avec et entre les jeunes et de tabler sur le soutien social et l'entraide.
\end{abstract}

La grossesse à l'adolescence est un phénomène que plusieurs qualifient de grave problème social. En Amérique du Nord, la grossesse à l'adolescence est associée à un ensemble de conséquences négatives, en particulier pour les adolescentes qui poursuivent leur grossesse et leurs enfants. Le but de la présente recherche est d'obtenir une meilleure connaissance du champ, ou contenu, de la représentation sociale de la grossesse à l'adolescence du point de vue d'adolescentes et d'adolescents, cela afin de repérer de nouvelles avenues de réflexion en ce qui a trait aux stratégies et contenus éducatifs dans ce domaine.

Au cours des 3 dernières décennies, le discours quant aux conséquences néfastes d'une grossesse, qualifiée de précoce, s'est modifié. Par exemple, à la fin des années 1970 et au début des années 1980, chercheurs et intervenants et intervenantes de la santé invoquaient les risques de complications médicales pour les jeunes mères et leurs enfants (Le Van, 1997). Depuis, des études recourant à des groupes témoins comparables ont montré que les adolescentes de plus de 15 ans recevant des soins prénatals adéquats n'étaient généralement pas plus à risque de complications médicales ou d'avoir des bébés prématurés ou de petit poids que les femmes plus âgées (Horwitz, Klerman, Kuo, \& Jekel, 1991; Ickovics et al., 2003; Le Van, 1997; Loto et al., 2004; Rodriguez \& Jimenez, 2001; Scholl, Hediger, \& Belsky, 1994; Smith \& Pell, 2001; Zimmerman, Tuttle, Kierrer, Parker, \& Caldwell, 2001). En fait, ces travaux indiquent que des conditions socio-économiques (telles que la pauvreté

Ce projet de recherche a été subventionné par le Conseil québécois de la recherche sociale. 
ou une moindre accessibilité aux soins de santé), sociofamiliales et scolaires, bien plus que l'âge, sont associées à ce type de complications (Bonell et al., 2003; Guilbert, Fortier, \& Mercier, 2004; Hillis et al., 2004; Manlove, 1998; Markham et al., 2003).

Le discours des chercheurs et intervenants et intervenantes s'est alors transformé et fait maintenant référence aux risques psychosociaux et socio-économiques associés à la grossesse dite précoce. Sur le plan psychosocial, des chercheurs avancent que la maternité à l'adolescence peut nuire à l'accomplissement de certains rôles propres à l'adolescence, celui d'élève par exemple (Clemmens, 2003); à la conciliation adolescence et maternité (Spear, 2004); à l'individuation et au développement de l'autonomie et de l'indépendance par rapport aux parents (Coley \& Chase-Lansdale, 1998); et à la santé psychologique à court et long termes (Bernazzani \& Bifulco, 2003; Omar, Martin, \& McElderry, 2001). En résulterait, chez ces jeunes mères, plus de détresse psychologique et une difficulté à remplir leur rôle parental.

Comme peu de travaux récents s'attardent aux risques psychologiques de la maternité à l'adolescence, Coley et Chase-Lansdale (1998) soutiennent qu'il serait important de mieux analyser le phénomène. Cournoyer (1995) mentionne également que les chercheurs se sont peu attardés à étudier les compétences dont peuvent faire preuve les jeunes mères. Certes, plusieurs études font ressortir des conséquences psychosociales négatives de la grossesse à l'adolescence sur les enfants, telles qu'un retard dans le développement cognitif, des problèmes de comportement à l'âge préscolaire et des taux plus élevés d'échecs scolaires et de délinquance à l'adolescence (Black et al., 2002; Furstenberg, Brooks-Gunn, \& Morgan, 1987; Haveman, Wolfe, \& Peterson, 1997; Miller, Bayley, Christensen Leavitt, \& Coyl, 2003). Cependant, une fois de plus, le statut socio-économique et la pauvreté apparaissent comme les déterminants les plus importants des problèmes de développement des enfants et des adolescents et adolescentes, plus que l'âge de la mère à la naissance.

Sur le plan des risques socio-économiques pour les mères adolescentes, chercheurs et intervenants et intervenantes font largement référence à leur faible scolarisation et à la pauvreté qui les attendent. Bien qu'un ensemble de résultats de recherche appuient le lien entre pauvreté et maternité à l'adolescence, des études viennent le nuancer, faisant ressortir que la pauvreté est déjà le lot de plusieurs adolescentes avant qu'elles ne deviennent enceintes (Brooks-Gunn, Guo, \& Furstenberg, 1993; Coley \& Chase-Lansdale, 1998; Cournoyer, 1995; Miller, Benson, \& Galbraith, 2001; Wilson, 1987; Zimmermann et al., 2001). Dans bien des cas, la pauvreté ne serait pas tant une conséquence de la grossesse, mais offrirait plutôt des conditions propices à sa survenue à l'adolescence. Par ailleurs, les quelques études longitudinales disponibles font ressortir que les effets à long terme de la maternité à l'adolescence ne sont pas aussi négatifs que les effets observés à court terme. Furstenberg et al. (1987) montrent que 17 ans après la naissance de leur enfant, plus des deux tiers (72\%) de mères adolescentes de milieu socio-économique faible qui ont eu accès à un suivi prénatal et postnatal occupaient un emploi. Horwitz et al. (1991) arrivent à des observations semblables pour des mères de milieu socio-économique faible qui avaient donné naissance à un enfant 20 ans plus tôt. Une proportion encore plus élevée de mères qui avaient également eu accès à un suivi prénatal et postnatal (82\%) étaient complètement indépendantes financièrement. Il semble que le soutien social, aussi bien formel qu'informel, soit un facteur clé dans leur adaptation, puisque ces mères faisaient face à des conditions de vie stressantes. 
Malgré ces résultats nuancés et encourageants en ce qui a trait à la grossesse à l'adolescence, les discours scientifique et populaire nord-américains demeurent généralement négatifs, parfois même stigmatisants. Les experts et expertes, aussi bien que le grand public, se posent les questions suivantes: Comment se fait-il qu'une proportion importante d'adolescentes deviennent enceintes à l'adolescence, alors que les moyens de contraception sont considérés accessibles? Pourquoi des jeunes filles, ou leur partenaire, seraient-elles enclines à poursuivre une grossesse à l'adolescence si la situation se présentait? Voilà autant de questions qui ont conduit l'équipe de recherche à étudier le champ de la représentation sociale de la grossesse à l'adolescence chez les adolescentes et les adolescents, à qui on donne peu l'occasion de se faire entendre sur le sujet.

Le champ d'une représentation correspond à l'ensemble des thèmes abordés quand il est question de l'objet de représentation. La question de recherche qui nous intéresse est celle-ci: Est-ce que les jeunes abordent les thèmes, ou dimensions, mentionnés plus haut, en partageant ces prises de position plutôt négative de la grossesse à l'adolescence? Le but de la recherche est d'obtenir une meilleure compréhension du champ de la représentation sociale de la grossesse à l'adolescence, du point de vue de jeunes, afin de repérer de nouvelles avenues de réflexion en ce qui a trait aux stratégies et contenus éducatifs dans ce domaine.

\section{CADRE CONCEPTUEL}

Le point de départ de l'approche théorique qui est privilégiée ici est que les comportements sont largement influencés par la représentation sociale d'un objet ou d'une situation (Abric, 1994; Doise, 1989; Moscovici, 1990). Ainsi, les pratiques des adolescentes et des adolescents, par exemple l'adoption ou non de certains comportements visant à prévenir la grossesse, seraient étroitement liées à la représentation de la grossesse à l'adolescence à laquelle adhèrent les jeunes. Conformément à la définition proposée par Abric (1989), par « représentation » il est question d' " un ensemble organisé d'opinions, d'attitudes, de croyances et d'informations se référant à un objet ou une situation. Elle est déterminée à la fois par le sujet lui-même (son histoire), par le système social et idéologique dans lequel il est inséré, et par la nature des liens que le sujet entretient avec ce système social » (p. 188). Ainsi, la représentation de la grossesse à l'adolescence n'est pas le fait purement individuel ou personnel d'un acteur solitaire. Elle est le résultat d'un processus complexe de construction sociale auquel participe trois types d'acteurs: l'acteur individuel (l'adolescente, l'adolescent), l'acteur intermédiaire immédiat (les groupes d'appartenance: famille, pairs) et l'acteur collectif (la société). La perspective adoptée ne signifie pas pour autant que les jeunes forment un groupe homogène. Au contraire, les représentations des jeunes peuvent révéler des prises de position variées, que nous souhaitons explorer.

Il s’agit d'abord de documenter le champ de la représentation de la grossesse à l'adolescence à travers le repérage des dimensions autour desquelles le discours des groupes de jeunes s'organise. Il s'agit ensuite de relever les prises de position dominantes à cet égard, ainsi que les prises de position divergentes ou, formulé autrement, celles qui confrontent les positions dominantes donc qui peuvent paraître déstabilisantes aux différents acteurs sociaux qui visent, consciemment ou non, le contrôle social. L'une de nos préoccupations, en tant que tenantes des grandes orientations de la psychologie communautaire (Dufort \& Guay, 2001; Rappaport, 1977; Rappaport \& Sidman, 2000), est que nos pratiques éducatives puissent 
s'inscrire dans une perspective de changement social plutôt qu'elles ne viennent alimenter le contrôle social. Le contrôle est actuellement omniprésent quand il est question de prévention des grossesses à l'adolescence, c'est-à-dire qu'il y a une forte propension à vouloir imposer la prise de position dominante, celle de la grossesse à l'adolescence comme grave problème social, sans trop tenir compte des autres prises de position. Cette propension peut s'avérer contre-productif lorsqu'il s'agit d'intervenir auprès des jeunes.

\section{MÉTHODOLOGIE}

\section{Population à l'étude et modalités de recrutement}

La recherche concerne des groupes d'adolescentes et d'adolescents, ainsi que des groupes d'intervenantes et d'intervenants travaillant auprès d'eux dans des écoles secondaires, des cliniques médicales ou de planification des naissances et dans des organismes communautaires (ex: maisons de jeunes). Sont rapportées ici uniquement les données recueillies auprès des groupes d'adolescentes et d'adolescents; d'autres résultats sont présentés ailleurs (Dufort, Boucher, Guilbert, Herrera, \& Saint-Laurent, 1999; Dufort, Guilbert, \& Saint-Laurent, 2000). Il nous importait de cerner le champ de la représentation des garçons aussi bien que celui des filles. Les garçons sont trop souvent mis de côté dans les études qui portent sur la grossesse à l'adolescence, ce qui peut laisser entendre que cela ne relève pas de leur responsabilité. Des filles et des garçons de 15 à 17 ans, ayant expérimenté ou non la grossesse à l'adolescence, participent à l'étude. Nous avons retenu les 15-17 ans car au moment de la réalisation de l'étude, l'âge moyen lors de la première relation sexuelle était d'environ 15,5 ans au Québec (Camirand, 1996). Ces jeunes sont issus de trois milieux socioéconomiques: un milieu faiblement nanti, un milieu moyennement nanti et un troisième très bien nanti.

Les jeunes ont été recrutés dans des écoles secondaires, mais aussi dans des maisons de jeunes et autres organismes communautaires de la communauté urbaine de Québec. Il paraît en effet important de rejoindre également les jeunes qui ne fréquentent pas ou peu l'école, ce qui permet de tenir compte de la diversité des milieux d'interaction dans lesquels ils évoluent.

Au total 150 participants et participantes répartis en 10 groupes (focus groups) d'adolescentes $(n=93)$ et 8 groupes d'adolescents $(n=57)$ sont rencontrés: $59 \%$ des jeunes proviennent de milieu faiblement nanti, $27 \%$ de milieu moyennement nanti et $14 \%$ de milieu très nanti. Leur âge moyen est de 16 ans. Par ailleurs, $71 \%$ des jeunes se disent sexuellement actifs; moins de $5 \%$ ont déjà été placés devant une grossesse. Cinq des focus groups ont été menés auprès de jeunes fréquentant des organismes de la communauté, tandis que les autres focus groups ont été réalisés dans les écoles.

\section{Méthode de collecte}

Les données ont été collectées à l’aide de la méthode du focus group. Une des particularités de cette méthode est d'utiliser explicitement l'interaction de groupe pour faciliter l'émergence de thèmes, d'idées qui seraient difficilement accessibles autrement (Krueger, 1994). Cette méthode n’a pas de visée consensuelle: elle favorise autant l'expression d'opinions semblables que contradictoires (Simard, 1989). Elle permet également aux leaders d'opinion de remplir le rôle qu'ils jouent habituellement dans leur milieu, tout en s'assurant que les autres participants et participantes puissent aussi émettre leurs opinions. Le focus group comporte certains 
avantages lorsqu'on le compare à l'entrevue individuelle. Ces avantages résident dans sa ressemblance avec les processus de formation d'opinions et de propagation d'information dans la vie quotidienne, puisque le focus group permet de cerner les représentations en situation de communication avec les autres (Albrecht, Johnson, \& Walther, 1993). En précisant que le focus group est un événement de communication au cours duquel l'interaction de l'individuel et du social peut être systématiquement observée, Millward (1995) souligne son utilité dans l'investigation des représentations sociales. Du point de vue de la psychologie communautaire, le focus group est également une méthode qui sert de première amorce à un dialogue entre les groupes d'acteurs concernés par une problématique, le dialogue étant un élément essentiel du changement social (Le Bossé \& Dufort, 2001).

\section{Procédure}

Dans le cadre de l'étude, les jeunes participants et participantes aux focus groups ont été répartis en fonction du sexe, du milieu socio-économique et du lieu privilégié d'interaction, soit l'école ou l'organisme communautaire. Les focus groups ont été réalisés par des animatrices formées et elles étaient secondées par un observateur qui se chargeait de l'enregistrement des discussions de groupe, ce qui assurait la représentation des deux sexes à l'intérieur de l'équipe de recherche. Le guide de discussion, prétesté auprès de deux groupes, était de type semi-structuré et consistait en des questions ouvertes sur les thèmes suivants: la grossesse à l'adolescence (sousthèmes: en quoi elle consiste, les réactions, les conséquences, les échanges entre jeunes, le désir de grossesse), sa prévention, les moyens de contraception et la double protection (mts/sida et grossesse non planifiée). Les questions étaient précédées d'une association de mots, ${ }^{1}$ mots qui par la suite faisaient l'objet de discussions. Les discussions ont été filmées et aussi enregistrées sur bandes audio.

Toutes les précautions pour assurer un consentement libre et éclairé de la part des participants et des participantes et la confidentialité des données ont été prises. Avant de participer à la recherche, les jeunes se sont vues expliquer les objectifs du projet, ils ont eu à lire et, s'ils consentaient à participer, à signer la formule de consentement. Le protocole de recherche ainsi que les formules de consentement éclairé ont reçu l'approbation du comité de déontologie de la recherche de l'Université Laval ainsi que du comité de déontologie médicale et pharmaceutique du Centre hospitalier universitaire de Québec (CHUQ).

\section{Analyse des données}

Le contenu intégral des discussions a été transcrit verbatim. Le recours au logiciel $N U D^{*} I S T$ a permis une gestion efficace des données textuelles. Une analyse de contenu qualitative a été effectuée selon la démarche proposée par L’Écuyer (1987) et a porté sur les groupes et non sur les participantes ou participants pris individuellement. Les étapes suivies sont les suivantes: constitution du corpus; lectures répétées du corpus; choix de l'unité d’analyse, dans ce cas-ci l’idée; création de catégories, selon un système mixte; codage des unités; procédures de validation de la catégorisation et du codage; analyse de premier degré menant à une première description; et analyse de second degré menant à une organisation signifiante des données. Plus précisément, l'analyse de premier degré permet de rendre compte, d'une part, des dimensions (regroupant différents thèmes) de la représentation sociale auxquelles les groupes font référence et, d'autre part, de la diversité des prises de position. L'analyse de second degré permet, pour sa part, l'élaboration d'une typologie à partir des dimensions et des prises de position. L'analyse de contenu 
effectuée sur les données ne vise pas à classer chaque participante ou participant selon l'une ou l'autre dimension ou selon les diverses prises de position possibles. Elle sert plutôt à cerner le champ de la représentation de la grossesse à l'adolescence et à dégager les principes organisateurs de ce champ à partir desquels des groupes de participantes et de participants peuvent prendre position.

\section{RÉSULTATS}

Mentionnons tout d'abord que, bien que le thème de la grossesse à l'adolescence intéresse beaucoup les groupes de jeunes de 15 à 17 ans, ces mêmes jeunes disent ne pas, sinon rarement, aborder le sujet entre eux. Les focus groups deviennent donc une occasion d'établir un dialogue entre les jeunes présents et entre chaque groupe de jeunes et l'équipe de recherche. Deuxièmement, pour la majorité des groupes de jeunes interviewés, la grossesse à l'adolescence relève de " l'accident », c'est-à-dire que si une grossesse survenait, il s’agirait généralement d’un événement inattendu, non planifié qui aurait habituellement pour cause un défaut de contraception (absence ou mauvaise utilisation). Troisièmement, la majorité des jeunes, du moins ceux et celles qui ont accepté de participer à la recherche, disent ne pas prendre la grossesse à l'adolescence à la légère. Les jeunes qui ont participé à la recherche sont généralement animé(e)s d'un questionnement éthique en ce qui a trait à l'issue de la grossesse, qu'ils fassent référence à sa poursuite ou à son interruption. Quatrièmement, tous les groupes de jeunes parlent abondamment des conséquences de la grossesse à l'adolescence; ils font alors référence à des aspects psychologiques et biologiques aussi bien qu'à des aspects économiques et sociaux.

La représentation sociale de la grossesse à l'adolescence s'organise autour de quatre dimensions: émotive, réflexive, psychobiologique et économico-sociale. Les groupes de jeunes prennent diverses positions envers ces dimensions qui laissent entrevoir leur questionnement éthique. On peut qualifier ces prises de position, à partir de leur discours, de négative, positive, ambivalente ou dynamique. À partir de ces dimensions et prises de position, nous avons construit une typologie des significations accordées par les groupes de jeunes à la grossesse à l'adolescence. Les types de signification identifiés sont (a) la grossesse-problème, (b) la grossesseprojet, (c) la grossesse-tension et (d) la grossesse-pouvoir. La désignation des différents types de signification sert à faciliter la description des résultats et à " faire image ». Il est important, cependant, de préciser qu'en général ces types de signification ne se présentent pas à l'état pur. De plus, les types de signification ne servent pas à classer des individus, mais plutôt à dégager différents points de vue au sujet des enjeux de la grossesse à l'adolescence entre les groupes et à l'intérieur des groupes.

\section{Une prise de position négative: la grossesse-problème}

Une participante d'un groupe de filles s'exclame:

Si je tombais enceinte, ça serait la fin du monde (F4-F1)! ${ }^{2}$

Un participant d'un groupe de garçons dit:

Surtout à notre âge, là t'arrives avec ça, bang! Tu sais, à 25 ans, ça serait cool, mais en ce moment, il y a un problème en quelque part (F6-G5).

Voilà deux affirmations, relevées, qui caractérisent la grossesse-problème. Pour les groupes de jeunes qui endossent cette prise de position, l'idée de vivre une telle expérience, si jeune et sans ressources financières, paraît inacceptable. C'est un événement catastrophique qui hypothèque généralement l'avenir de l'adolescente. 
Pour ces jeunes, les enjeux de la mise à terme d'une grossesse représentent un grave problème sur plusieurs plans: émotif, réflexif, psychobiologique et économico-social.

Sur le plan émotif, l'annonce d'une grossesse causerait à ces jeunes une surprise ou un choc: "Je capoterais », "Je badtriperais ", "Je virerais folle », " Je ferais une attaque cardiaque ", "Je pète une coche », "T'es vraiment désordonnée » sont autant d'extraits de leurs discours qui caractérisent leur position négative. Ces réactions proviennent d'abord d'une évaluation défavorable des circonstances qui entoureraient la grossesse. De plus, des adolescentes et des adolescents croient qu'une grossesse non planifiée, non désirée et précoce viendrait perturber leur plan d'avenir et nuire à leur réputation. Ainsi, les jeunes croient que les réactions du partenaire, des parents, de la famille, des amis et amies ou des pairs seraient négatives. L'adolescente enceinte et son partenaire craignent la désapprobation de leur entourage et les termes souvent employés pour caractériser cette situation sont la panique, la tristesse, la honte, le rejet et l'isolement. Selon des groupes de jeunes, c'est surtout dans les familles où la valorisation des études et de la carrière est importante que la grossesse à l'adolescence est inacceptable et doit, par conséquent, être résolue par l'avortement.

Sur le plan réflexif, conscient(e)s qu'ils sont de plus en plus exposés à faire l'expérience d'une grossesse accidentelle, étant donné la libéralisation et la précocité des relations sexuelles, certain(e)s jeunes estiment qu'il ne peut y avoir questionnement de la grossesse à l'adolescence sans tenir compte de la morale ou de l'éthique. Ils croient que l'adolescente enceinte et son partenaire sont tenus de prendre une décision rationnelle quant à la résolution de la grossesse. Il s’avère que ce ne sont pas tant les conséquences de l'interruption de la grossesse qui suscitent un questionnement chez les jeunes porteurs d'une position négative que les enjeux liés à sa poursuite. Généralement, les adolescentes et les adolescents se considèrent trop jeunes pour avoir un enfant. Les groupes de jeunes qui ont intériorisé le modèle dominant du passage à l'âge adulte ont tendance à considérer la grossesse à l'adolescence comme non souhaitable car elle transgresse les normes sociales s'y rapportant.

Une autre conséquence ajoutée par les jeunes au bilan négatif de la grossesseproblème est la forte probabilité que la mère adolescente se retrouve seule. Ainsi, selon eux, l’enfant sera pénalisé parce qu'il n’aura pas de père. Également selon leurs principes, avoir un bébé serait une décision irresponsable. Ces adolescentes et adolescents envisagent l'avortement comme une solution efficace et acceptable. Des adolescentes sont convaincues que leurs parents ou leur partenaire les inciteraient à se faire avorter; néanmoins elles insistent sur le fait que ce sont elles qui prendraient la décision.

Sur le plan psychobiologique, les jeunes qui entrevoient la grossesse à l'adolescence de manière problématique déplorent le stress que celle-ci génère habituellement de même qu'ils suspectent les séquelles psychologiques graves pour l'adolescente enceinte. Elle peut devenir dépressive et négliger sa santé ou son bien-être. Parmi les jeunes qui conçoivent la grossesse comme un problème, les adolescentes semblent plus préoccupées par les conséquences physiques ou biologiques tandis que les adolescents insistent davantage sur les changements d'affects ou d'humeurs de l'adolescente enceinte. La prise de poids et la transformation corporelle, surtout à l'adolescence où l'idéal de la minceur est très présent, sont généralement jugées défavorables. Dans leur logique, le corps d'une adolescente ne doit pas porter le stigmate de la maternité. Disant avoir observé ou entendu parler, dans leur entourage, 
des femmes enceintes qui souffraient de malaises ou de problèmes de santé, d'autres expriment leur crainte face aux symptômes suivants: varices, enflures, maux de dos.

Enfin, sur le plan économico-social, ces jeunes se représentent de façon très négative, parfois même de manière dramatique, les conditions matérielles et financières dans lesquelles vivent habituellement les mères adolescentes. En effet, comme l'adolescente enceinte est souvent abandonnée par son partenaire, elle doit assumer seule sa grossesse et, éventuellement, les responsabilités à l'égard de l'enfant à naître. Elle devient alors très dépendante du soutien de ses parents. Il peut arriver aussi qu'elle subisse le rejet des parents et qu'elle doive se débrouiller seule. Les jeunes estiment que l'une des conséquences les plus malheureuses et défavorables pour l'adolescente enceinte est l'obligation de quitter le foyer familial. Un autre grand problème serait que la mère adolescente manque d'argent. Les jeunes croient aussi que les adolescentes sont souvent victimes de préjugés et de discrimination, tant de la part de certain(e)s jeunes que de certain(e)s adultes.

\section{Une prise de position positive: la grossesse-projet}

Voici des extraits tirés du discours des jeunes qui caractérisent, cette fois, la grossesse-projet. Une adolescente déclare:

Quand tu sais que tu attends un enfant, me semble que tu es contente (F3-F4).

Un adolescent s'exprime dans le même sens:

Bien, moi, la première réaction, j'ai dit elle va sûrement le donner. Moi, je vais le prendre, le bébé. Je me suis dis je vais être papa; j’étais content. Crime, je vais avoir un enfant (F13-G3).

La grossesse-projet se fonde généralement sur une image symbolique du bébé qui représente la vie, l'amour, bref, le bonheur. Pour les filles ou les garçons qui endossent l'idée d'une grossesse-projet, sur le plan émotif, la grossesse accidentelle est perçue comme un événement heureux. Leurs propos laissent entendre que, d'une part, ils n'auraient pas planifié la grossesse, mais que, d'autre part, ils seraient néanmoins contents de garder l'enfant même après l'avoir conçu par accident. Ainsi, contrairement à des stéréotypes bien ancrés, certains adolescents se montrent aussi intéressés et motivés que des adolescentes par l'enfant qu'ils auraient conçu même de façon involontaire. Si l'adolescente enceinte et son partenaire estiment qu'ils sont prêts à s'engager dans une relation de couple stable avec l'espoir de fonder une famille, ils vont s'en réjouir. Ils ne mettent pas en doute la maturité ni la capacité de leur partenaire à assumer de façon responsable le rôle de parent. De plus, si la grossesse survient à un âge plus avancé de l'adolescence, c'est-à-dire vers 17 ou 18 ans, ils croient que c'est plus facile de l'accepter que si elle arrivait trop tôt, vers l'âge de 12 ans, par exemple. Un autre facteur important qui faciliterait cette acceptation serait la bonne communication existant entre les jeunes et leurs parents. Des adolescentes sont convaincues que leurs parents ou ceux de leur partenaire auraient une réaction positive si elles leur annonçaient leur grossesse.

En ce qui a trait à la dimension réflexive, les jeunes qui partagent une position favorable à l'égard de la grossesse et de la maternité ou de la paternité à l'adolescence adhèrent à un système de valeurs communes axées sur la valorisation de l'enfant, perçu comme symbole de la vie, de l'amour. En conséquence, pour ces jeunes, l'avortement est condamnable parce qu'il signifie un meurtre ou un crime. Ils pensent que la grossesse à l'adolescence peut représenter un projet de vie significatif pour des adolescentes qui sont en quête de réalisation personnelle ou de reconnaissance sociale. 
À l'encontre d'un certain discours qui tend à discréditer l'ensemble des mères adolescentes, certain(e)s jeunes mettent plutôt l'accent sur leur maturité et leur capacité à assumer des responsabilités parentales quand ils font référence à la dimension psychologique. En ce qui concerne la dimension biologique, si certain(e)s jeunes déplorent les transformations estimées inesthétiques qui marquent le corps d'une femme enceinte, d'autres, en revanche, évoquent plutôt sa beauté. La " grosse bedaine » devient alors le symbole du nouveau statut social et les coups de pied du bébé, le symbole de la vie.

Sur le plan économico-social, la grossesse-projet correspond à la possibilité de quitter la famille d'origine et d'acquérir un statut social. En faisant abstraction du père de l'enfant et sans avoir franchi les étapes dites normales du passage à l'âge adulte, la grossesse deviendrait alors une stratégie pour obtenir un revenu et un statut. Certains mentionnent des cas d'adolescentes qui ont réussi dans la vie, après avoir eu un enfant. De plus, il semble que la grossesse à l'adolescence n'est pas toujours vécue comme un drame familial puisque l'événement peut susciter de la compréhension et du soutien de la part des parents.

\section{Une prise de position ambivalente: la grossesse-tension}

L'ambivalence face à la grossesse à l'adolescence est fondée sur un discours dans lequel les conséquences négatives et positives coexistent. Dans cette perspective, la grossesse représente une situation sans issue favorable:

Je serais contente, mais je me dirais que je suis trop jeune (F1-F3).

Bien, tu sais, dans le fond, ça a deux côtés, un bon puis un mauvais. Il y a du positif mais il y a du négatif. Là, je serais content c'est un enfant. Mais, après ça, quand j’y repenserais, je verrais tous les côtés négatifs de la chose (F11-G4).

D’une part, la grossesse paraît positive parce qu'elle est perçue comme un idéal qui se justifie sur le plan personnel; elle représente un projet de vie souhaitable et valorisant. D'autre part, elle est considérée négative parce qu'elle est jugée condamnable sur le plan médical et social. Le conflit entre deux univers symboliques en compétition, la légitimité d'un projet personnel et la croyance en la nécessaire conformité aux normes sociales semble brouiller la vision du monde de certain(e)s jeunes. Ce conflit paraît refléter l'opposition entre des logiques différentes qui structurent les discours: rationalité versus affectivité, conformité versus déviance, initiative individuelle versus adhésion aux valeurs du groupe.

Les jeunes qui s'expriment de manière ambivalente face à la grossesse à l'adolescence disent éprouver à la fois des sentiments négatifs (de peur, d'angoisse, de désarroi, d'insécurité) et des sentiments positifs (de désir de réalisation, de joie, de bonheur):

Moi, je serais heureuse, mais j'aurais peur aussi (F10-F4).

Sur le plan réflexif, ces jeunes semblent vivre une certaine paralysie devant les décisions à prendre et les actions à poser. L'analyse des échanges sur les solutions envisagées montre que les jeunes ne les considèrent pas à la légère. Pour eux, l'avortement, par exemple, n'est pas une décision facile à prendre sur le plan moral ou religieux. Par ailleurs, il leur paraît difficile de transgresser les normes du modèle dominant de l'entrée dans la vie adulte qui, bien entendu, exclut le projet d'un bébé à l'adolescence. En outre, des adolescentes se disent incertaines quant à l'idée d'annoncer ou non la survenue de la grossesse à leur partenaire ou à leurs parents. En effet, d'un côté, elles craignent leur réaction négative, de l'autre, elles souhaitent leur aide ou leur soutien. 
Quand il est question des dimensions psychobiologique ou économico-sociale, les jeunes qui expriment une position ambivalente reprennent à la fois les arguments évoqués pour décrire les réactions négatives face à la grossesse-problème (transformations inesthétiques du corps, avenir gâché) et les réactions positives face à la grossesse-projet (beauté de la bedaine, acquisition d'un statut social).

\section{Une prise de position dynamique: la grossesse-pouvoir}

Ces jeunes qui endossent une position dite dynamique ont une représentation de la grossesse à l'adolescence qui dépasse largement la conception d'un problème social, ainsi que la notion de pensée magique liée au désir d'enfant. En effet, il est plutôt question d'un certain pouvoir que veulent s'approprier des jeunes sur leur vie ou sur leur environnement. En somme, ils réclament le droit de décider par euxmêmes:

Si les deux partenaires sont consentants puis qu'ils en ont parlé avec leurs parents puis qu'ils voudraient. T'as les moyens, bien, pourquoi t'en fais pas un flo? C'est quoi qui l'interdit? Tu sais, c'est la société qui dit les flos, on fait ça plus tard. Tu sais, les flos plus tard, c'est cave! (F16-G6).

La question qu'ils se posent est celle-ci: est-ce que les grossesses tardives ne seraient pas aussi problématiques que les grossesses précoces?

Ces jeunes manifestent une résistance aux normes sociales et au modèle dominant du passage à l'âge adulte:

Oui, mais un bébé, je peux l'avoir quand ça me tente. Là, c'est sûr qu'aux yeux du monde, ils pensent qu'il faut être adulte parce qu'ils pensent que t'as de l'argent, t'as ci, t'as ça. Quand t'es jeune, bien, t'es immature, t'es pas capable de faire ci, de faire ça. Bien oui, les maudits adultes, ils ont des préjugés (F16-G5).

Des adolescentes estiment que le jeune âge ne constitue pas toujours un obstacle à la maternité; certaines évoquent l'expérience positive de leur mère. Ces filles et garçons se disent animés d'un sentiment de pouvoir.

Les jeunes qui estiment que la poursuite d'une grossesse peut devenir un projet d'avenir mettent l'accent sur la nécessité de réfléchir aux exigences de la parentalité. Ils pensent qu'il est nécessaire également de ne pas se soucier de la désapprobation de l'entourage. Selon eux, il faut assumer ses choix peu importe ce que les gens peuvent dire ou penser. Ces adolescents et adolescentes ont un regard critique sur chacune des autres positions: négative, positive et ambivalente. Il s'agit, pour eux, d'une prise en compte de la complexité des choix à effectuer plutôt que la réduction prétendument rationnelle, affective ou conflictuelle de celle-ci. Contrairement à la position ambivalente qui est caractérisée par la difficulté à résoudre la situation de grossesse, la position dynamique met plutôt l'accent sur la capacité des jeunes à prendre conscience des contradictions et à mieux y faire face. Ils cherchent à se construire une identité à partir d'un choix libre et conscient entre plusieurs possibilités.

Ces jeunes remettent en question les valeurs d'une société dominée par l'argent et la réussite sociale:

Oui, l'âge, puis le monde pense beaucoup matériel. Tu sais, dans le fond, tu peux ne pas avoir beaucoup d'argent puis être heureux quand même (F15-F1).

Pour les comprendre, il ne faut pas analyser leurs conditions matérielles d'existence, mais plutôt leur conception du bonheur. En effet, selon certain(e)s, la société s'est transformée, modernisée, mais elle ne traite pas mieux les femmes adultes qui sont sur le marché du travail et qui ont des enfants. Ainsi, pour cer- 
tain(e)s, la poursuite de la grossesse à l'adolescence peut devenir une stratégie pour vaincre l'exclusion sociale.

\section{DISCUSSION}

Le but de la recherche était d'obtenir une meilleure compréhension du champ de la représentation sociale de la grossesse à l'adolescence du point de vue des jeunes, cela afin de repérer de nouvelles avenues de réflexion en ce qui a trait aux stratégies et contenus éducatifs dans ce domaine. Les représentations sociales permettent de mieux comprendre les liens que les jeunes entretiennent avec le système social. Certain(e)s jeunes semblent vouloir adhérer et se conformer au modèle dominant du passage à l'âge adulte, qui veut qu'après avoir effectué des études et commencé une vie professionnelle, les jeunes s'établissent avec un partenaire et fondent une famille (Galland, 2001). D'autres, au contraire, se disent prêts à agir à l'encontre de ces normes sociales. D'autres jeunes encore sont ambivalent(e)s face à des normes qui, trop souvent, les placent devant des choix paradoxaux. Enfin, des jeunes remettent carrément en question ces normes et renvoient les adultes à leurs contradictions. La grossesse à l'adolescence fait référence à diverses prises de position au travers desquelles nous devinons des acteurs actifs dans la quête du sens à donner à la grossesse, à sa poursuite ou à son interruption. Leurs propos sont d'ailleurs marqués par les conflits entre la raison et la passion, entre l'individualisme et le collectivisme, entre le conformisme et la marginalité, entre le déterminisme et le pouvoir d'agir.

La plupart des jeunes interviewé(e)s semblent avoir intégré une large part des messages des adultes qui les entourent (Jaccard, Dodge, \& Dittus, 2003), avec tout ce que cela comporte de contradictions, d'incertitudes et de conflits. Ceux et celles qui remettent en question les messages dominants endossent des prises de position que nous avons qualifiées de déstabilisantes parce qu'elles renvoient au désarroi, au sentiment d'impuissance ou à la colère que des intervenants et intervenantes expriment quand ils sont confrontés à ces jeunes qui conçoivent la grossesse à l'adolescence comme un projet possible ou comme une source de pouvoir. Certains intervenants ou intervenantes se disent déstabilisés, ne sachant plus quelles actions entreprendre (Dufort et al., 2000). Lors d'une entrevue de recherche, une intervenante suggérait l'action suivante comme solution au problème social que représentait selon elle la grossesse à l'adolescence: «Si j'étais le ministre de la santé, je ferais passer une loi pour obliger les adolescentes à se faire avorter. »

À la lumière de ces constats, il s’avérerait pertinent que les contenus éducatifs prennent en compte la complexité du phénomène de la grossesse à l'adolescence en intégrant l'ensemble des prises de position des jeunes. En outre, parce que la plupart des jeunes sont animé(e)s d'une réflexion critique, il serait judicieux que les stratégies éducatives se fondent sur un dialogue ouvert avec et entre les jeunes. Ce dialogue devrait aller au-delà d'un discours alarmiste, qui porte habituellement sur les causes et les conséquences négatives de la grossesse à l'adolescence, puisque les jeunes les connaissent bien. Comme Freire (1970) l'a montré, le dialogue est à la base d'un processus de conscientisation, de développement du pouvoir d'agir et de réfléchir qui facilite la clarification de ses propres prises de position, qui permet de prendre ses distances par rapport aux influences sociales en vue d'effectuer des choix plus informés.

À travers leur discours, les jeunes ont d'ailleurs très bien souligné l'importance des diverses influences sociales, parfois soutenantes, d'autres fois absentes, voire même néfastes, dans leur rapport à la grossesse à l'adolescence. En fait, le soutien 
social apparaît comme un élément clé, aussi bien dans le discours des jeunes que dans les écrits scientifiques (Clemmens, 2003; Spear, 2004). Il s’avère donc primordial de le mettre à profit dans les interventions éducatives. La grossesse à l'adolescence n’a pas avantage à être traitée comme un " problème " individuel appartenant à certain(e)s jeunes. Les interventions peuvent mettre un accent particulier sur le développement de l'entraide en plus du dialogue (Dryfoos, 1998). Les stratégies d'entraide permettent de sensibiliser les jeunes aux prises de position des autres, ce qui peut favoriser une plus grande acceptation de la diversité, prévenir la marginalisation et, par extension, créer un environnement soutenant (Levine, 1998). Par exemple, il peut s'agir, lors d'une intervention en classe, de favoriser le développement du soutien émotif (e.g., empathie, écoute active), du soutien cognitif (e.g., connaissance des ressources en vue d'orienter les pairs) et du soutien instrumental (e.g., partage des coûts de la contraception entre partenaires). Plusieurs études montrent bien que la présence de soutien social et sa qualité entrent souvent en ligne de compte dans la prévention des grossesses accidentelles (Miller et al., 2001), en plus de faire la différence entre une grossesse menant à des échecs et une grossesse source de croissance (Coley \& Chase-Lansdale, 1998).

\section{CONCLUSION}

En ce qui a trait aux limites, lors de cette étude nous avons pu rejoindre uniquement les jeunes qui voulaient bien participer à la recherche. Cette situation a pu entraîner un biais de sélection, les jeunes les plus volubiles ou se sentant davantage concernés étant peut-être plus susceptibles d'accepter l'invitation. Il faut néanmoins mentionner que l'équipe de recherche a fait des efforts pour recruter des jeunes dans divers milieux, notamment des jeunes de la rue à qui on donne rarement la parole. Par ailleurs, chaque méthode de collecte de données comporte des limites. Le recours à des focus groups peut faire en sorte que certains thèmes, la violence dans les relations amoureuses par exemple, ne soient pas abordés. Nous sommes conscientes de cette limite et c'est pourquoi nous suggérons que la recherche se poursuive en ayant recours à d'autres méthodes de collecte et en faisant appel à d'autres groupes concernés. En outre, la présente recherche visait à cerner le champ de la représentation de la grossesse à l'adolescence et à repérer diverses prises de position; elle ne portait pas sur l'analyse systématique des processus d'ancrage et d'objectivation. D'autres études seront nécessaires à l'exploration de ces processus, mais la démarche devait commencer par une exploration du champ de la représentation de la grossesse à l'adolescence.

Mentionnons pour conclure que cette étude présente la particularité d'avoir été menée auprès de filles et de garçons, ce qui permet de mettre en évidence le fait que la grossesse peut relever de la responsabilité autant des garçons que des filles. En outre, contrairement à plusieurs études dans le domaine de la grossesse à l'adolescence, celle-ci a été menée auprès de jeunes qui n’ont pas nécessairement été placé(e)s devant une situation de grossesse, ce qui évite d'attribuer aux jeunes en général des observations faites auprès de populations cliniques. Enfin, un autre aspect original de cette recherche réside certainement dans le fait qu'elle a permis de faire émerger des prises de position variées face à la grossesse à l'adolescence et de remettre en question la prise de position dominante. 


\title{
NOTES
}

1. L'association était suscitée par la question suivante: «Quels mots ou images vous viennent spontanément en tête lorsque je dis grossesse à l'adolescence? »

2. Chaque citation est identifiée au focus group (F pour « focus group », suivi de son numéro d'identification) où elle a été exprimée et à la personne qu'il l'a formulée (F pour fille, $G$ pour garçon).

\begin{abstract}
This study was undertaken to gather information on the social representations of teenage pregnancy among adolescents, aged between 15 and 17. Eighteen focus groups were conducted among 150 boys and girls. The data were subjected to a qualitative content analysis. Results show that youths did not form homogeneous groups. The points of view expressed gave rise to 4 dimensions (emotive, reflexive, psychobiological, economic-social) and 4 positions (negative, positive, ambivalent and dynamic). From these dimensions and positions, 4 representations of teenage pregnancy were identified: pregnancy as a problem, pregnancy as a project, pregnancy as a source of tension, and pregnancy as a source of power. This study illustrates the importance of educative strategies such as going beyond alarmist preventive messages, opening dialogue with and between youngsters, and promoting social support and mutual aid.
\end{abstract}

\section{RÉFÉRENCES}

Abric, J.C. (1989). L’étude expérimentale des représentations sociales. Dans D. Jodelet (dir.), Les représentations sociales (pp.187-219). Paris: Presses Universitaires de France.

Abric, J.C. (1994). Pratiques sociales, représentations sociales. Dans J.C. Abric (dir.), Pratiques sociales et représentations (pp. 217-251). Paris: Presses Universitaires de France.

Albrecht, T.L., Johnson, G.M., \& Walther, J.B. (1993). Understanding communication processes in focus groups. Dans D.L. Morgan (dir.), Successful focus groups: Advancing the state of the art (pp. 51-64). Thousand Oaks, CA: Sage Publications.

Bernazzani, O., \& Bifulco, A. (2003). Motherhood as a vulnerability factor in major depression: The role of negative pregnancy experiences. Social Science \& Medicine, 56(6), 1249-1260.

Black, M.M., Papas, M.A., Hussey, J.M., Hunter, W., Dubowithz, H., Kotch, J.B., et al. (2002). Behavior and development of preschool children born to adolescent mothers: Risk and 3generation households. Pediatrics, 109(4), 573-580.

Bonell, C.P., Strange, V.J., Stephenson, J.M., Oakley, A.R., Copas, A.J., Forrest, S.P., et al. (2003). Effect of social exclusion on the risk of teenage pregnancy: Development of hypotheses using baseline data from a randomised trial of sex education. Journal of Epidemiology and Community Health, 57(11), 871-876.

Brooks-Gunn, J., Guo, G., \& Furstenberg, F.F., Jr. (1993). Who drops out of and who continues beyond high school? A 20-year follow-up of Black urban youth. Journal of Research on Adolescence, 3(3), 271-294.

Camirand, J. (1996). Enquête sociale et de santé 1992-1993: un profil des enfants et des adolescents québécois. Québec, QC: Ministère de la Santé et des Services sociaux, Santé Québec.

Clemmens, D. (2003). Adolescent motherhood: A meta-synthesis of qualitative studies. American Journal of Maternal/Child Nursing, 28(2), 93-99.

Coley, R.L., \& Chase-Lansdale, L.P. (1998). Adolescent pregnancy and parenthood: Recent evidence and future directions. American Psychologist, 53(2), 152-166.

Cournoyer, M. (1995). Maternité précoce: un passage inédit à l’âge adulte. P.R.I.S.M.E., 5(2-3), 266-287.

Doise, W. (1989). Attitudes et représentations sociales. Dans D. Jodelet (dir.), Les représentations sociales (pp. 220-238). Paris: Presses Universitaires de France. 


\section{REVUE CANADIENNE DE SANTÉ MENTALE COMMUNAUTAIRE}

Dryfoos, J.G. (1998). Safe passage: Making it through adolescence in a risky society: What parents, schools, and communities can do. New York: Oxford University Press.

Dufort, F., Boucher, K., Guilbert, É., Herrera, M., \& Saint-Laurent, L. (1999, août). Teenage pregnancy and its prevention: Social representations of teenagers and practitioners. Communication par affiche présentée à la Convention of the American Psychological Association, Boston.

Dufort, F., \& Guay, J. (2001). Agir au cœur des communautés: la psychologie communautaire et le changement social. Sainte-Foy, QC: Presses de l’Université Laval.

Dufort, F., Guilbert, É., \& Saint-Laurent, L., en collaboration avec la Direction de la santé publique de Québec. (2000). La grossesse à l'adolescence et sa prévention: au-delà de la pensée magique. Québec, QC: Conseil québécois de la recherche sociale (CQRS).

Freire, P. (1970). Pedagogy of the oppressed. New York: Herder and Herder.

Furstenberg, F.F., Jr., Brooks-Gunn, J., \& Morgan, P. (1987). Adolescent mothers and their children in later life. Family Planning Perspectives, 19(4), 142-151.

Galland, O. (2001). Sociologie de la jeunesse. Paris: Amond Colin.

Guilbert, É., Fortier, M., \& Mercier, P. (2004). Grossesse à l'adolescence 1998-2000 et interruption volontaire de grossesse 1998-2002 dans la région de la Capitale nationale. Québec, QC: Direction régionale de santé publique, Agence de développement des réseaux locaux de services de santé et de services sociaux de la Capitale nationale.

Haveman, R.H., Wolfe, B., \& Peterson, E. (1997). Children of early childbearers as young adults. Dans R.A. Maynard (dir.), Kids having kids: Economic costs and social conesquences of teen pregnancy (pp. 257-284). Washington, DC: Urban Institute Press.

Hillis, S.D., Anda, R.F., Dube, S.R., Felitti, V.J., Marchbanks, P.A., \& Marks, J.S. (2004). The association between adverse childhood experiences and adolescent pregnancy, long-term psychosocial consequences and fetal death. Pediatrics, 113(2), 320-327.

Horwitz, S.M., Klerman, L.V., Kuo, H.S., \& Jekel, J.F. (1991). School-age mothers: Predictors of long-term educational and economic outcomes. Pediatrics, 87(6), 862-868.

Ickovics, J.R., Kershaw, T.S., Westdahl, C., Rising, S.S., Klima, C., Reynolds, H., et al. (2003). Group prenatal care and preterm birth weight: Results from a matched cohort study at public clinics. Obstetrics \& Gynecology, 102(5), 1051-1057.

Jaccard, J., Dodge, T., \& Dittus, P. (2003). Maternal discussions about pregnancy and adolescents' attitudes toward pregnancy. Journal of Adolescent Health, 33(2), 84-87.

Krueger, R.A. (1994). Focus groups: A practical guide for applied research (2e édition). Thousand Oaks, CA: Sage Publications.

Le Bossé, Y., \& Dufort, F. (2001). Le pouvoir d'agir (empowerment) des personnes et des communautés: une autre façon d'intervenir. Dans F. Dufort (dir.) \& J. Guay (codir.), Agir au cour des communautés: la psychologie communautaire et le changement social (pp. 75115). Sainte-Foy, QC: Presses de l'Université Laval.

L’Écuyer, R. (1987). L’analyse de contenu: notions et étapes. Dans J.P. Deslauriers (dir.), Les méthodes de recherche qualitative (pp. 49-66). Sillery, QC: Presses de l’Université du Québec.

Le Van, C. (1997). Les grossesses adolescentes: drame réel ou incongruité sociale? Revue de sociologie et d'anthropologie, 3, 139-167.

Levine, M. (1998). Prevention and community. American Journal of Community Psychology, 26(2), 189-206.

Loto, O.M., Ezechi, O.C., Kalu, B.K., Loto, A., Ezechi, L., \& Ogunniyi, S.O. (2004). Poor obstetric performance of teenagers: Is it age- or quality of care-related? Journal of Obstetrics and Gynecology, 24(4), 395-398.

Manlove, J. (1998). The influence of high school drop out and school disengagement on the risk of school-age pregnancy. Journal of Research on Adolescence, 8(2), 187-220.

Markham, C.M., Tortolero, S.R., Escobar-Chaves, S.L., Parcel, G.S., Harrist, R., \& Addy, R.C. (2003). Family connectedness and sexual risk-taking among urban youth attending alternative high schools. Perspectives on Sexual and Reproductive Health, 35(4), 174-179.

Miller, B.C., Bayley, B.K., Christensen Leavitt, S.C., \& Coyl, D.D. (2003). Adolescent pregnancy and childbearing. Dans G.R. Adams et M.D. Berzonsky (dir.), Blackwell handbook of adolescence (pp. 415-449). Oxford, Royaume-Uni: Blackwell Publishing. 


\section{LES REPRÉSENTATIONS SOCIALES DE LA GROSSESSE À L’ADOLESCENCE}

Miller, B.C., Benson, B., \& Galbraith, K.A. (2001). Family relationships and adolescent pregnancy risk: A research synthesis. Developmental Review, 21, 1-38.

Millward, L.J. (1995). Focus groups. Dans G.M. Breakwell \& C. Fife-Schaw (dir.), Research methods in psychology (pp. 274-292). London: Sage Publications.

Moscovici, S. (1990). The origins of social representations: A response to Michael. New Ideas in Psychology, 8(3), 383-388.

Omar, H.A., Martin, C., \& McElderry, D. (2001). Screening for depression in adolescents: Association with teen pregnancy. Journal of Pediatric and Adolescent Gynecology, 14(3), 129-133.

Rappaport, J. (1977). Community psychology: Values, research, and action. New York: Holt, Rinehart and Winston.

Rappaport, J., \& Sidman, E. (2000). Handbook of community psychology. New York: Kluwer Academic/Plenum.

Rodriguez, M.A., \& Jimenez, M.A. (2001). Epidemiological assessment of the influence of socio-family factors in adolescent pregnancy. European Journal of Epidemiology, 17(7), 653-659.

Scholl, T.O., Hediger, M.L., \& Belsky, D.H. (1994). Prenatal care and maternal health during adolescent pregnancy: A review and meta-analysis. Journal of Adolescent Health, 15, 444456.

Simard, G. (1989). Animer, planifier et évaluer l'action: guide d'utilisation de la méthode du groupe focus. Montréal: Mondia Éditeurs.

Smith, G.C.S., \& Pell, J.P. (2001). Teenage pregnancy and risk of adverse perinatal outcomes associated with first and second births: Population based retrospective cohort study. BMJ (British Medical Journal), 323(7311), 476-480.

Spear, H.J. (2004). A follow-up case study on teenage pregnancy: « Havin’ a baby isn’t a nightmare, but it’s really hard ». Pediatric Nursing, 30(2), 120-125.

Wilson, W.J. (1987). The truly disadvantaged: The inner city, the underclass, and public policy. Chicago: University of Chicago Press.

Zimmerman, M.A., Tuttle, L., Kierrer, E., Parker, E., \& Caldwell, C.H. (2001). Psychosocial outcomes of urban African American adolescents born to teenage mothers. American Journal of Community, 29(5), 779-805. 